\title{
Expression of NOB1 Gene and Its Clinical Significance in Ovarian Cancer
}

\author{
Ning LIU ${ }^{1}$, Shun-qing ZHOU ${ }^{1}$ and Yang LIN $^{1, a, *}$ \\ ${ }^{1}$ Department of Obstetrics and Gynecology, The Second Hospital, Jilin University, \\ \#218 Ziqiagn Street, Nanguan District, Changchun 130041, China \\ alinyang1155@163.com \\ ${ }^{*}$ Corresponding author
}

Keywords: Ovarian Cancer, NOB1, Quantitative RT-PCR.

\begin{abstract}
Purposes] To explore the correlation between mRNA expression of NOB1 gene in tissues from ovarian cancer, normal ovary, benign ovarian tumor. [Methods] 50 samples of ovarian cancer as the experimental group, 20 samples of normal ovarian tissues and 50 samples of benign ovarian tumor as control groups. Expression of NOB1 gene was evaluated by RT-PCR. [Results]The mRNA Expression of NOB1 gene in ovary cancer tissues was significantly higher than that in normal ovarian tissues $(\mathrm{P}<0.01)$, while there was no significant difference between normal ovary and innocent ovary. High level of NOB1 mRNA expression was correlated to tumor size and degree of cell differentiation $(\mathrm{P}<0.05)$, but not significantly correlated to pathology type, pathological stage of operation and age $(\mathrm{P}>0.05)$. [Conclusions]Compared to normal ovary and benign ovarian tumori, mRNA expression of NOB1 gene was higher in ovarian cancer which indicates that mRNA expression level of NOB1 gene in ovary may be correlated to the occurrence and development of ovarian cancer.
\end{abstract}

\section{Introduction}

Ovarian cancer, one of the most common gynecological cancers, is a major cause of gynecological tumors deaths [1]. In 2002, 204449 new cases and 124860 deaths were estimated worldwide according to IARC statistics [2], and the five-year survival rates are still less than 50\% [3]. Ovarian cancer represents a great clinical challenge in gynecological tumors, for the reason that most patients are asymptomatic until the disease has metastasized and two-thirds of the patients are diagnosed with advanced-stage disease [4]. The incidence of ovarian cancer in China has been in a significantly rising trend [5]. The 26S proteasome, an ATP-dependent protease in eukaryotic cells, is a $2.5-\mathrm{MDa}$ molecular machine mainly consisted of $20 \mathrm{~S}$ core particle structure and 19S regulatory subunit, which is responsible for hydrolyzing ubiquitin protein. The $20 \mathrm{~S}$ core is hollow and provides an enclosed cavity in which proteins are degraded. The 19S regulatory subunit contains multiple ATPase active sites and ubiquitin binding sites; which are responsible for recognizing polyubiquitinated proteins and transfers them to the catalytic core. RNA-binding proteins are key molecules in post-transcriptional regulation of gene expression which regulate cell function by involving in all aspects of RNA metabolism from RNA splicing, sequence editing, RNA transport, RNA stabilization to translation control [13].

NOB1 (NIN1/RPN12 binding protein 1 homolog) is one subunit of $26 \mathrm{~S}$ proteasome which plays crucial role in protease function and RNA metabolism. Human NOB1 gene is composed of nine exons and eight introns and located at chromosome 16q22.1. 
NOB1 protein is an evolutionarily conserved protein, which comprises an $\mathrm{N}$-terminal PIN domain and a C-terminal zinc ribbon domain and is expressed mainly in liver, lung and

spleen [6-8]. Abnormal expression of NOB1 gene in leukemia tumor has been detected in previous studies. Recently, it has been shown that NOB1 is an important regulator of the tumorigenic properties of human hepatocellular carcinoma and could be used as a candidate therapeutic target in human hepatocellular carcinoma [9]. These results suggest that NOB1 may involved in other tumors, however, as a subunit of $26 \mathrm{~S}$ proteasome, little information on expression of NOB1 and its role in other tumors is available. To our knowledge, the correlation between expression of NOB1 gene and ovarian cancer risk is still not well understood [10]. The aim of the present study was to investigate mRNA expression of NOB1 gene in ovarian cancer and assess its role in the occurrence and development of ovarian cancer.

\section{Materials and Methods}

\section{Patients and Samples}

The fifty specimens of ovarian cancer were collected from patients who underwent surgery for primary ovarian cancer at the department of gynecology, second affiliated hospital of Jilin university, from December 2014 to December 2015. All the patients provided with detailed information. Twenty specimens of normal ovarian tissues were collected from patients who were diagnosed with cervical cancer or endometrial carcinoma and subjected to ovary resection. Pathological examination was performed to confirm that ovarian metastasis was not happened. Fifty specimens of benign ovarian tumor were collected from patients who were diagnosed with ovary serous cystadenoma or ovarian mucinous cystadenoma and subjected to ovary resection. The patients with benign ovarian were confirm not to be metastatic tumor of ovary by pathological examination. All the patients gave signed informed consent, and the present study was approved by the institutional ethics review boards. The size of ovarian specimens was about $1.5 \mathrm{~cm} \times 1.5 \mathrm{~cm}$. The specimens were immediately frozen in liquid nitrogen and then transferred to refrigerator $\left(-80^{\circ} \mathrm{C}\right)$ until processing for RNA analysis.

The age of the fifty patient with ovarian cancer ranges from 35 to 70 (38 patients below 60 years of age, 12 patients over 60 years of age). Fifty specimens were collected from patients with ovarian cancer of different pathologic type, among which 19 cases were serous carcinoma, 15 cases were mucinous carcinoma, 8 cases were endometrial carcinoma, and 8 cases were other types. Respects to pathologic stage, 19 cases were early ovarian cancer ( 7 cases of stage I, 12 cases of stage II), 31 cases were advanced ovarian cancer ( 22 cases of stage III, 9 cases of stage IV). Respects to differentiation degree, 31 cases were poorly differentiated and 19 cases were well-differentiated cancer or moderately differentiated cancer. Informed written consent was obtained from all subjects, and the study was approved by the Ethics Committee of second affiliated hospital of Jilin university, China.

\section{RNA Isolation and cDNA Synthesis}

Total RNA was isolated from frozen ovarian tissue using TRIzol reagent (Invitrogen Corp., Carlsbad, CA) according to the manufacturer's protocol and as described in the online supplement. The quality and quantity of the RNA was verified by the presence of two discrete electropherogram peaks corresponding to the $28 \mathrm{~S}$ and $18 \mathrm{~S}$ rRNA at a 
ratio approaching 2:1.For all samples, $5 \mu \mathrm{g}$ of total RNA was treated with $3 \mathrm{U}$ of DNase I to remove contaminating genomic DNA.

\section{Reverse Transcription-PCR Quantification of Mrna Expression}

In order to assess the mRNA expression of NOB1 gene in ovaries, $\beta$-actin gene was selected for reference gene. Oligonucleotide primers for $\beta$-actin and NOB1 genes were designed according to GenBank. The primers for NOB1 and $\beta$-actin gene are given below. The first primer is the forward PCR primer and the second is the reverse PCR primer. NOB1: 5'-ATCTGCCCTACAAGCCTAAAC-3'; 5'-TCCTCCTCCTCCTCCTCAC-3'. The size of PCR production was 180 bp. $\beta$-actin: 5'-GGCGGCACCACCATGTACCCT -3'; 5'-AGGGGCCGGACTCGTCATACT-3'. The size of PCR production was $150 \mathrm{bp}$.

Two step methods for real-time fluorescence quantitative RT-PCR was applied. $\Delta \mathrm{CT}$ value is calculated for each sample as the difference between the CT values for the NOB1 gene and the $\beta$-actin gene in each sample. The $\Delta \Delta C T$ value is the difference between the $\Delta \mathrm{CT}$ values of ovary cancer tissues and the samples of benign ovarian tumor. The fold-change in NOB1 gene expression is equal to $2-\Delta \Delta \mathrm{CT}$ if the PCR replication efficiency for all genes is 100 percent.

\section{Statistical Analysis}

To calculate the statistical differences among groups, the statistical package SPSS16.0 was used for all analysis. Student's test was used to determine the significant difference of mRNA expression of NOB1 gene in different groups. The data were expressed as mean values \pm standard deviations (SD). Statistical significance was accepted when $P<0.05$, and statistical significantly difference was accepted when $P<0.01$.

\section{Results}

\section{Expression of NOB1 Gene in Ovarian Cancer Tissues, Normal Ovarian Tissues and Benign Ovarian Tumor}

The amplification curve of PCR production is presented in Figure 1. The mRNA expression of NOB1 gene in ovarian cancer tissues, normal ovarian tissues and benign ovarian tumor is shown in Table 1. The results showed that NOB1 gene was expressed in ovarian cancer tissues, normal ovarian tissues and benign ovarian tumor. The $2^{-\Delta \Delta C T}$ method was used to analyses the data and normal ovary was as control ${ }^{[11]}$. The results showed that mRNA expression of NOB1 gene in ovary cancer tissues is significantly higher than that in normal ovaries $(P<0.01)$ and the difference is about 10.63 times, however, there is no significant difference in normal ovarian tissues and benign ovarian tumor $(P>0.05)$. When PCR reaction completes, melting curve of each sample will be automatically generated according to the fluorescence value during degeneration. The melting curve is shown in figure 2 . As shown in figure 2 , there is no miscellaneous peak and abnormal broadening main peak in melting curve of primers for NOB1 and $\beta$-actin gene, which indicates that there was no nonspecific amplification caused by pollution and primer dimmer and so on. 


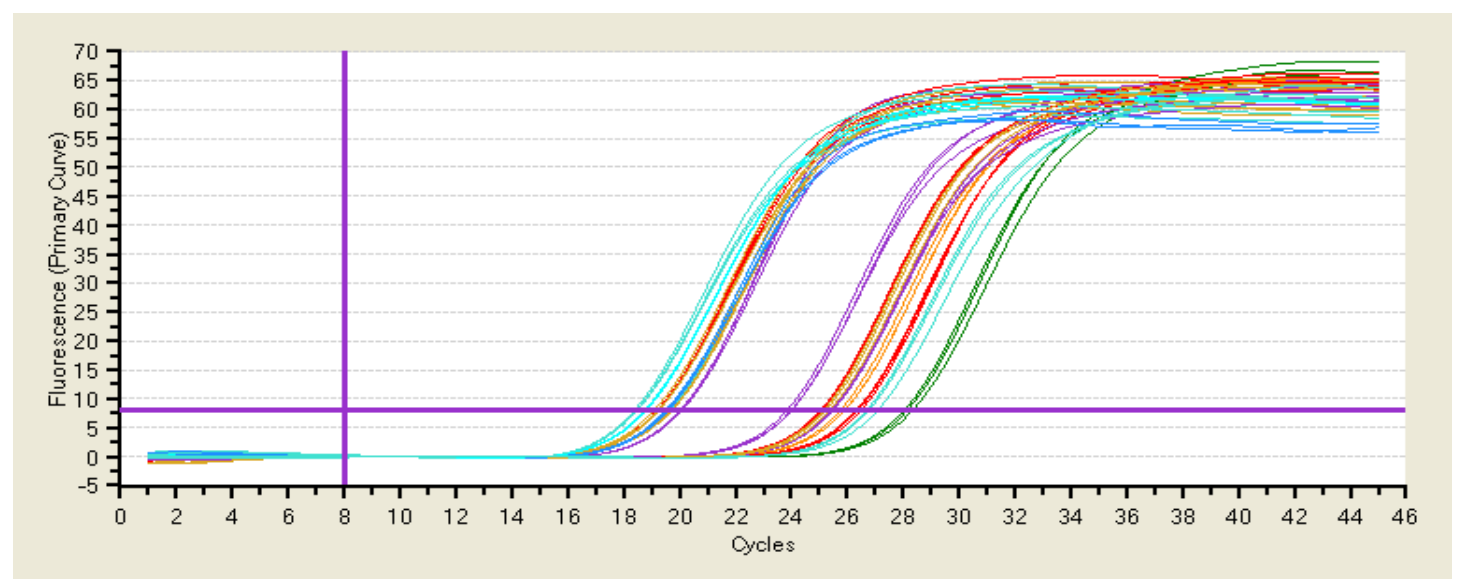

Figure1. Amplification curve of NOB1 gene in real-time PCR.

Table 1. mRNA expression of NOB1 gene in tissues from ovarian cancer, normal ovarian and benign

\begin{tabular}{cccc}
\multicolumn{4}{c}{ ovarian tumor $($ Mean \pm SD) } \\
\hline & case & NOB1 mRNA $\Delta C T$ & $2-\Delta \Delta C T$ \\
\hline varian cancer tissues & 50 & $11.17 \pm 2.48$ & $10.63 \pm 2.68^{* *}$ \\
benign ovarian tumor & 50 & $14.69 \pm 2.89$ & $0.93 \pm 2.93$ \\
normal ovarian tissues & 20 & $14.58 \pm 3.27$ & $1.00 \pm 0.37$ \\
\hline
\end{tabular}

Note: $\Delta C T=C T(N O B 1)-C T(\beta$-actin) $\quad \Delta \Delta C T=$ Mean of $\Delta C T$ (control group) $-\Delta C T$ (samples)

$* * \mathrm{p}<0.01$, which indicates that there is significant difference in mRNA expression of NOB1 gene between normal ovarian and varian cancer tissues.

Actin

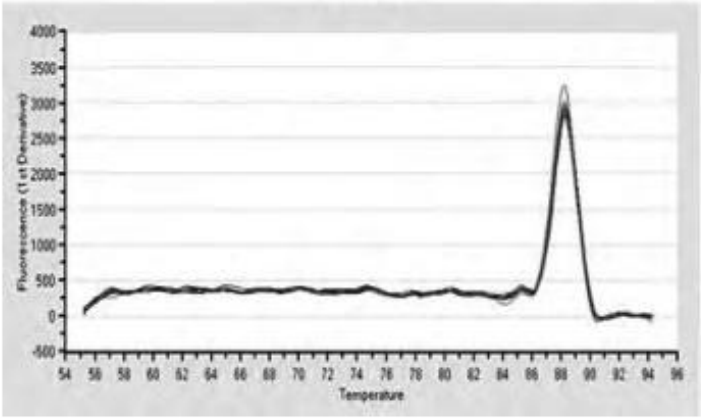

NOB1

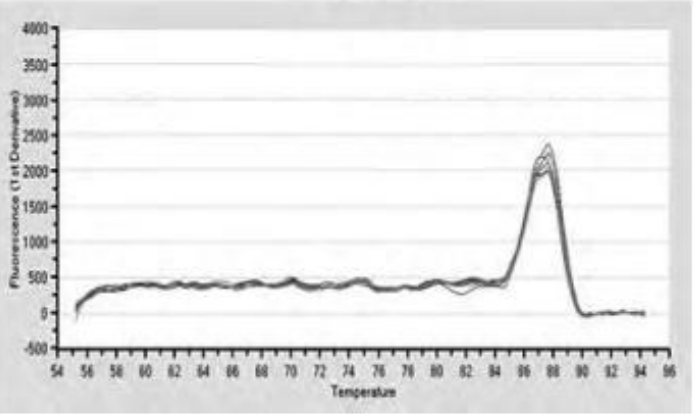

Figure 2. Melting curve of primers for $\beta$-actin and NOB1

\section{Expression of NOB1 Gene in Ovarian Cancer Tissues with Different Clinical-Pathologic Features}

Expression of NOB1 gene in ovarian cancer tissues with different clinical-pathologic features is shown in table 2. In the present study, positive expression was considered when the ratio of average expression amount of NOB1 gene in ovarian cancer tissues to that in normal ovary is more than 2 , and the negative expression was confirmed when the ratio is less than or equal to 2 . As shown in table 2 , the positive expression in the 50 samples of ovarian cancer is $64 \%$ (32/50). High level of NOB1 mRNA expression was correlated to tumor size and degree of cell differentiation $(\mathrm{P}<0.05)$, but not significantly correlated to pathology type, pathological stage of operation and age $(\mathrm{P}>0.05)$. 
Table 2 Comparison of mRNA expression of NOB1 gene in ovarian cancer tissues with different. clinical pathological characteristics $(\mathrm{Mean} \pm \mathrm{SD})$

\begin{tabular}{|c|c|c|c|}
\hline category & case & positive NOB1 & $P$ value \\
\hline \multicolumn{4}{|l|}{ Age(year) } \\
\hline$\leq 60$ & 17 & 11 & \multirow{2}{*}{$>0.05$} \\
\hline$>60$ & 33 & 21 & \\
\hline \multicolumn{4}{|l|}{ Size of tumor $(\mathrm{cm})$} \\
\hline$\leq 3$ & 23 & 10 & \multirow{2}{*}{$<0.05^{*}$} \\
\hline$>3$ & 27 & 22 & \\
\hline \multicolumn{4}{|l|}{ Pathological type } \\
\hline serous carcinoma & 19 & 12 & \multirow{4}{*}{$>0.05$} \\
\hline mucinous carcinomas & 15 & 11 & \\
\hline endometrial carcinoma & 8 & 4 & \\
\hline Other type & 8 & 5 & \\
\hline \multicolumn{4}{|l|}{ Degree of cell differentiation } \\
\hline well or moderate differentiation & 19 & 10 & \multirow[t]{2}{*}{$<0.05^{*}$} \\
\hline poor differentiation & 31 & 22 & \\
\hline \multicolumn{4}{|l|}{ Stage of tumor } \\
\hline Early stage & 19 & 13 & \multirow[t]{2}{*}{$>0.05$} \\
\hline Advanced stage & 31 & 19 & \\
\hline
\end{tabular}

\section{Discussion}

Ovarian cancer is the cancer with the highest mortality rate among gynecologic tumor. Due to the insidious onset of the disease and the lack of effective detection means, two-thirds of patients with ovarian cancer will develop to be the advanced stage when definite diagnosis were made. Although combinations of surgical and cytotoxic therapy were applied to the patient, nearly $90 \%$ of them will develop again to the diseases ${ }^{[12]}$. Therefore, it is great important to identify novel prognostic and predictive biomarkers for ovarian cancer and fully understand the molecular mechanism of the occurrence and development of ovarian cancer which may eventually lead to the development of novel and more effective treatment method for ovarian cancer.

The $26 \mathrm{~S}$ proteasome is an ATP-dependent protease in eukaryotic cells, which is a 2.5-MDa molecular machine and consisted of $20 \mathrm{~S}$ core particle structure and $19 \mathrm{~S}$ regulatory subunit. Nob1p, which interacts with Rpn12, a subunit of the 19S regulatory particle (RP) of the yeast $26 \mathrm{~S}$ proteasome, was firstly identified by yeast two-hybrid screening and discovered to be correlated to formation and maturation of 26S proteasome ${ }^{[13]}$. Moreover, Nob1p involves in the regulation of rRNA by combining with larger and smaller subunit of ribosome ${ }^{[14,15]}$. Zhang et al (2005) cloned NOB1 gene, the homologous gene of human Nob1p. NOB1 gene encodes protein of 412 amino acids and the length of NOB1 mRNA is 1733 bp. NOB1 comprises an N-terminal PIN domain and a C-terminal zinc ribbon domain (ZRD1) ${ }^{[16]}$. It has been shown that ZRD1 can suppress cell proliferation and stimulate cell 
apoptosis by suspending cell cycle to G1 stage. As a subunit of $26 \mathrm{~S}$ proteasome, NOB1 is expressed in various tissues and possesses relative function of $26 \mathrm{~S}$ proteasome.

Results of the present study showed that NOB1 was expressed in ovarian cancer tissues, normal ovarian tissues and benign ovarian tumor. The mRNA expression of NOB1 gene in ovarian cancer tissues was significantly higher than that in normal ovarian tissues and benign ovarian tumor. The high level of NOB1 expression may favor its regulatory role on transformation of G1 stage of cell cycle and suppress cell proliferation and development of tumor.

Results of the present study showed that NOB1 gene mRNA was highly expressed in early and advanced ovarian cancer tissues and there is no significant difference between the two stages. This result suggests that NOB1 gene contributes to the occurrence and development of ovarian cancer. Furthermore, high level of mRNA expression of NOB1 gene was found in ovarian tumor whose diameter is more than or equal to $5 \mathrm{~cm}$. This result indicates that NOB1 gene could be used as diagnostic biomarker for ultrasonic examination of patients with ovarian cancer (diameter $\geq 5 \mathrm{~cm}$ ). NOB1 gene also may provide evidence for diagnosis of ovarian cancer.

The mRNA expression of NOB1 gene in tissues from ovarian cancer patients with different differentiation degree was also investigated in the present study. The results showed that mRNA expression of NOB1 gene in well-differentiated or moderately differentiated cancer tissues was significantly lower than that in poorly differentiated cancer tissues $(\mathrm{P}<0.05)$. This result suggests that NOB1 gene is correlated to the development of ovarian cancer. However, the difference of mRNA expression of NOB1 gene needs to be confirmed with large sample experiment. There was no significant difference in mRNA expression of NOB1 gene among epithelial ovarian cancer, different pathological type of epithelial ovarian cancer and other types of ovarian cancer, which suggests that NOB1 gene is not correlated to pathological type of ovarian cancer.

In conclusion, high level of mRNA expression of NOB1 gene in ovarian cancer tissues is beneficial to suppression of protein synthesis and the negative regulation of cell cycle, which decreases tumor cell proliferation and suppresses the development of ovarian cancer. High level of mRNA expression of NOB1 gene in ovarian tumor (diameter $\geq 5 \mathrm{~cm}$ ) and poorly differentiated cancer tissues indicates that NOB1 can suppress the development of tumor by regulating transformation of G1 stage of cell cycle with zinc ribbon domain. Therefore, NOB1 gene could be used as a diagnostic biomarker for ovarian cancer and provide evidence for diagnosis of ovarian cancer and enlightening insight to the development of molecular therapy for ovarian cancer.

\section{Acknowledgement}

This research was financially supported by Norman Bethune Program of Jilin University [2015213].

\section{References}

[1] Jayson, G. C.; Kohn, E. C.; Kitchener, H. C.; Ledermann, J. A. Ovarian cancer. Lancet 384:1376-1388; 2014.

[2] Faggad A, Darb-Esfahani S, Wirtz R, Sinn B, Sehouli J, Könsgen D, et al. Topoisomerase II $\alpha$ mRNA and protein expression in ovarian carcinoma: correlation with clinicopathological factors and prognosis. Mod Pathol 2009; 22(4):579-88. 
[3] Siegel, R. L.; Miller, K. D.; Jemal, A. Cancer statistics, 2016. CA Cancer J Clin 66:7-30; 2016.

[4] Orfanelli, T.; Jeong, J. M.; Doulaveris, G.; Holcomb, K.; Witkin, S. S. Involvement of autophagy in cervical, endometrial and ovarian cancer. Int J Cancer 135:519-528; 2014.

[5] Mizushima, N.; Levine, B.; Cuervo, A. M.; Klionsky, D. J. Autophagy fights disease through cellular self-digestion. Nature 451:1069-1075; 2008.

[6] Wang, H.; Li, P.; Zhao, B. Knockdown of NOB1 expression by RNAi inhibits cellular proliferation and migration in human gliomas. Gene 528:146-153; 2013.

[7] Liu, K.; Gu, M. M.; Chen, H. L.; You, Q. S. NOB1 in non-small-cell lung cancer: expression profile and clinical significance. Pathol Oncol Res 20:461-466; 2014.

[8] Liu, G.; Shen, D.; Jiao, L.; Sun, Y. Nin one binding protein expression as a prognostic marker in prostate carcinoma. Clin Transl Oncol 16:843-847; 2014.

[9] Zhang, X.; Zhang, D.; Qu, F.; Hong, Y.; Cao, J.; Pan, X.; Li, L.; Huang, Y.; Huang, H.; Yin, L.; Chen, L.; Ren, J.; Wang, Z.; Xu, D.; Cui, X. Knockdown of NOB1 expression inhibits the malignant transformation of human prostate cancer cells. Mol Cell Biochem 396:1-8; 2014.

[10] Lin Y, Peng S, Yu H et al. RNAi-mediated downregulation of NOB1 suppresses the growth and colony-formation ability of human ovarian cancer cells. Med Oncol.

[11] Livak KJ, Schmittgen TD. Analysis of Relative Gene Expression Data Using Real-Time Quantitative PCR and the 2- $\Delta \Delta$ CT Method. Methods 2001; 25: 402-408.

[12] Park, M. A.; Choi, K. C. Effects of 4-nonylphenol and bisphenol A on stimulation of cell growth via disruption of the transforming growth factor-beta signaling pathway in ovarian cancer models. Chem Res Toxicol 27:119-128; 2014.

[13] Tone Y \& Toh-E A. Nob1p is required for biogenesis of the $26 \mathrm{~S}$ proteasome and degraded upon its maturation in Saccharomyces cerevisiae. Gene Dev 2002; 16: 3142-3157.

[14] Vierstra RD. The ubiquitin/26S proteasome pathway, the complex last chapter in the life of many plant proteins. Trends Plant Sci 2003; 8: 135-142.

[15] Alessandro F, David T, MensurD. PIN domain of Nob1p is required for D-site cleavage in 20S pre-rRNA. RNA 2004, 10:1698-1701.

[16]Y. Zhang, J. Ni, G. Zhou, et al. Cloning expression and characterization of the human NOB1 gene. Mol Biol Rep 2005; 32(3):185-189. 